\title{
Intellectual property (IP)
}

Public Domain

\section{Source}

Open Research Glossary

A legal term that refers to creations of the mind. Examples of intellectual property include music, literature, and other artistic works; discoveries and inventions; and phrases, symbols, and designs. 